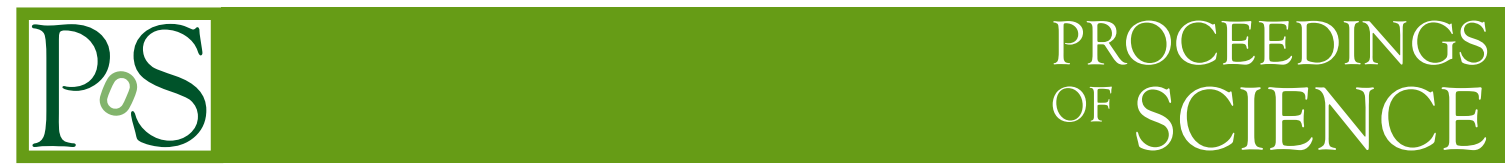

\title{
Implications of CKM Unitarity
}

\section{William J. Marciano*}

Brookhaven National Laboratory

Upton, New York 11973

E-mail: marciano@bnl.gov

Three generation CKM Unitarity in the Standard Model predicts $\left|V_{u d}\right|^{2}+\left|V_{u s}\right|^{2}+\left|V_{u b}\right|^{2}=1$. Nuclear, $K_{\ell 3}$ and $b \rightarrow u$ decays currently give $\left|V_{u d}\right|^{2}+\left|V_{u s}\right|^{2}+\left|V_{u b}\right|^{2}=0.9991(8)$. The good agreement between theory and experiment can be used to constrain "new physics" effects at the tree and quantum loop level. Examples considered are: exotic muon decays, heavy quark or lepton mixing, high scale induced 4 fermion operators and additional $Z^{\prime}$ gauge bosons.

Kaon International Conference

May 21-25, 2007

Laboratori Nazionali di Frascati dell'INFN

${ }^{*}$ Speaker. 
Unitarity of the bare (unrenormalized) Cabibbo-Kobayashi-Maskawa (CKM) [1] $3 \times 3$ quark mixing matrix $V_{i j}^{0}, i=u, c, t j=d, s, b$ implies the orthonormal tree level relations

$$
\sum_{i} V_{i j}^{0 *} V_{i k}^{0}=\sum_{i} V_{j i}^{0 *} V_{j k}^{0}=\delta_{j k}
$$

Standard Model quantum loop effects are important and corrected for such that eq. 11 continues to hold at the renormalized level [2]. That prescription generally involves normalization of all charged current semileptonic amplitudes relative to the Fermi constant

$$
G_{\mu}=1.166371(6) \times 10^{-5} \mathrm{GeV}^{-2}
$$

obtained from the precisely measured (recently improved) muon lifetime [3]

$$
\tau_{\mu}=\Gamma^{-1}\left(\mu^{+} \rightarrow e^{+} v_{e} \bar{v}_{\mu}(\gamma)\right)=2.197019(21) \times 10^{-6} \mathrm{sec}
$$

In all processes, Standard Model $\mathrm{SU}(3)_{C} \times \mathrm{SU}(2)_{L} \times \mathrm{U}(1)_{Y}$ radiative corrections are explicitly accounted for [曰].

Of particular interest here is the first row constraint

$$
\left|V_{u d}\right|^{2}+\left|V_{u s}\right|^{2}+\left|V_{u b}\right|^{2}=1
$$

An experimental deviation from that prediction would be evidence for "new physics" beyond Standard Model expectations in the form of tree or loop level contributions to muon decay and/or the semileptonic processes from which the $V_{i j}$ are extracted. Of course, if eq. ( $($ ) is respected at a high level of certainty, it implies useful constraints on various "new physics" scenarios.

In the case of $V_{u d}$, its value is extremely well determined from superallowed nuclear beta decays $\left(0^{+} \rightarrow 0^{+}\right.$transitions) which are theoretically pristine to a high level of certainty [5]. Employing the (slightly) updated [6] $f t$ values in table 1 , I find the average

$$
\left|V_{u d}\right|=0.97377(10)_{\exp }(15)_{\text {nucl }}(19)_{\mathrm{RC}}=0.97377(26)
$$

where the uncertainties stem from experiment (primarily), nuclear theory and radiative corrections respectively 河. The good agreement among the nine different nuclei in table 1 , over a large range in $Z$, suggests that nuclear theory corrections are well under control and fairly represented by the error on $V_{u d}$.

The central value in eq. (5) is the same as in the last published survey [7] and very close to a conference value $(0.97378(27))$ recently reported by John Hardy [8]. It appears unlikely that $V_{u d}$ will shift much beyond the range in eq. (5). Nevertheless, a more definitive determination, without nuclear uncertaintites, would be welcome and is in principle possible using (planned) precise measurements of the neutron lifetime, $\tau_{n}$, and the decay asymmetry parameter, $g_{A} \equiv G_{A} / G_{V}$, via [1]

$$
\left|V_{u d}\right|^{2}=\frac{4908.7(1.9) \mathrm{sec}}{\tau_{n}\left(1+3 g_{A}^{2}\right)}
$$

The value of $V_{u s}$ is best obtained from $K_{\ell 3}$ decays $(K \rightarrow \pi \ell v, \ell=e, \mu)$. Flavianet [9] global fits to the neutral and charged $K_{\ell 3}$ decay rates give 


\begin{tabular}{ccc}
\hline \hline Nucleus & $f t(\mathrm{sec})$ & $V_{u d}$ \\
\hline${ }^{10} \mathrm{C}$ & $3039.5(47)$ & $0.97381(77)(15)(19)$ \\
${ }^{14} \mathrm{O}$ & $3043.3(19)$ & $0.97368(39)(15)(19)$ \\
${ }^{26} \mathrm{Al}$ & $3037.4(10)$ & $0.97396(22)(15)(19)$ \\
${ }^{34} \mathrm{Cl}$ & $3049.8(10)$ & $0.97415(23)(15)(19)$ \\
${ }^{38} \mathrm{~K}$ & $3051.1(10)$ & $0.97404(26)(15)(19)$ \\
${ }^{42} \mathrm{Sc}$ & $3046.9(10)$ & $0.97328(30)(15)(19)$ \\
${ }^{46} \mathrm{~V}$ & $3050.4(10)$ & $0.97285(32)(15)(19)$ \\
${ }^{50} \mathrm{Mn}$ & $3045.5(15)$ & $0.97372(40)(15)(19)$ \\
${ }^{54} \mathrm{Co}$ & $3048.4(11)$ & $0.97373(40)(15)(19)$ \\
\hline Weighted Ave. & & $0.97377(10)(15)(19)$ \\
\hline \hline
\end{tabular}

Table 1: Values of $V_{u d}$ implied by various precisely measured superallowed nuclear beta decays. The $f t$ values are taken from Savard et al. updated with more recent measurements of $Q$ values and half-lives [6]. Uncertainties in $V_{u d}$ correspond to 1) nuclear structure and $Z^{2} \alpha^{3}$ uncertainties added in quadrature with the $f t$ error, 2) a common error assigned to nuclear Coulomb distortion effects, and 3) a common uncertainty in the radiative corrections from quantum loop effects [7]. Only the first error is used to obtain the weighted average.

$$
f_{+}(0)\left|V_{u s}\right|=0.21668(45)
$$

where the form factor $f_{+}(0)$ deviates from 1 due to small second order SU(3) breaking. One generally employs the classic result of Leutwyler and Roos [10], $f_{+}(0)=0.961(8)$ which leads to $\left|V_{u s}\right|=0.2255(19)$. That value seems to be confirmed by lattice gauge theory studies [11, 12]. A recent [12] effort gave relatively small uncertainties

$$
f_{+}(0)=0.9609(51)
$$

From that result and eq. (五), one finds

$$
\left|V_{u s}\right|=0.2255(13)
$$

It is, however, worth noting that more recent chiral perturbation theory studies [13] find the larger $f_{+}(0)=0.974(12)$ which leads to a smaller $\left|V_{u s}\right|=0.2225(28)$ and, as we shall see, a potential violation of unitarity. That smaller value is supported by extractions of $V_{u s}$ from tau decays [14] and $K_{\mu 2}$ decays [15]; but, in all cases the errors are too large to be anything but suggestive.

Using the (rather negligible) $\left|V_{u b}\right|^{2} \simeq 1.5 \times 10^{-5}$ in conjunction with eqs. (5) and (9) leads to

$$
\left|V_{u d}\right|^{2}+\left|V_{u s}\right|^{2}+\left|V_{u b}\right|^{2}=0.9991(5)_{V_{u d}}(6)_{V_{u s}}=0.9991(8)
$$

The rather good agreement with unitarity provides an impressive confirmation of Standard Model radiative corrections (at about the 40 sigma level!). It can be used to constrain "new physics" effects which would manifest themselves as a deviation from 1, i.e. what would appear to be an apparent breakdown of unitarity. 
I will give several examples of the utility eq. (10) provides for constraining "new physics". Each case is considered in isolation, i.e. it is assumed that there are no accidental cancellations. Also, note, if the larger $f_{+}(0)=0.974(12)$ is used [13], it leads to the interesting deviation $\left|V_{u d}\right|^{2}+$ $\left|V_{u s}\right|^{2}+\left|V_{u b}\right|^{2}=0.9977(13)$. I will not consider that possibility here.

\section{i) Exotic Muon Decays}

If the muon can undergo decay modes beyond the Standard Model $\mu^{+} \rightarrow e^{+} v_{e} \bar{v}_{\mu}$ and its radiative extensions, those exotic decays will contribute to the muon lifetime. That would mean that the "real" Fermi constant, $G_{F}$, is actually smaller than the value in eq. (2) and we should be finding

$$
\left|V_{u d}\right|^{2}+\left|V_{u s}\right|^{2}+\left|V_{u b}\right|^{2}=1-B R(\text { exotic muon decays) }
$$

A unitarity sum below 1 could be interpreted at possible evidence for such decays. Alternatively, eq. (10) provides at (one-sided) $95 \% \mathrm{CL}$

$$
B R(\text { exotic muon decays })<0.0022
$$

That is, of course, not competitive with, for example, the direct bound $B R\left(\mu^{+} \rightarrow e^{+} \gamma\right) \lesssim 1 \times 10^{-11}$. However, for decays such as $\mu^{+} \rightarrow e^{+} \bar{v}_{e} v_{\mu}$ (wrong neutrinos), eq. (12) is about a factor of 5 better than the direct constraint [16] $B R\left(\mu^{+} \rightarrow e^{+} \bar{v}_{e} v_{\mu}\right)<0.012$. That is interesting for possible future neutrino factories where the neutrino beams originate from muon decays. If such a decay were to exist, it would provide a background to neutrino oscillations.

Another way to illustrate the above constraint is to extract the Fermi constant assuming the validity of CKM unitarity without employing muon decay. Semileptonic decays then give

$$
G_{F}=1.1658(5) \times 10^{-5} \mathrm{GeV}^{-2} \quad \mathrm{CKM} \text { Unitarity }
$$

which is in fact the second best determination of $G_{F}$, after eq. (2). The comparison between $G_{\mu}$ in eq. (2) and $G_{F}$ in eq. (13) is providing the constraints on "new physics".

ii) Heavy Quarks and Leptons

As a second example, consider the case of new heavy quarks or leptons that couple to the ordinary 3 generations of fermions via mixing [2]. For a generic heavy charge $-1 / 3 D$ quark from a 4 th generation, mirror fermions, $\mathrm{SU}(2)_{L}$ singlets etc., one finds at the one-sided $95 \% \mathrm{CL}$

$$
\left|V_{u D}\right| \leq 0.05
$$

Considering that $\left|V_{u b}\right| \simeq 0.004$, such an indirect constraint does not seem to be very stringent. In the case of heavy neutrinos with $m_{N}>m_{\mu}$, one finds

$$
\left|V_{\mu N}\right| \lesssim 0.02
$$




\section{iii) Four Fermion Operators}

If there are induced dim. 6 four fermion operators of the form

$$
\frac{2 \pi}{\Lambda^{2}} \bar{u} \gamma_{\mu} d \bar{e}_{L} \gamma^{\mu} v_{e}
$$

where $\Lambda$ is a high effective mass scale due to compositeness, leptoquarks, excited $W^{*}$ bosons (e.g. extra dimensions) or even heavy loop effects, they will interfere with the Standard Model beta decay amplitudes. Depending on the sign of the interference, one finds at one-sided 95\%CL

$$
\begin{array}{ll}
\Lambda>19 \mathrm{TeV} & \text { (destructive) } \\
\Lambda>44 \mathrm{TeV} & \text { (constructive) }
\end{array}
$$

Similar constraints (reversed) apply to new 4 fermion lepton operators that contribute to $\mu^{+} \rightarrow$ $e^{+} v_{e} \bar{v}_{\mu}$. Of course, in some cases there can be a cancellation between semileptonic and purely leptonic effects.

The high scale bounds in eq. (17) apply most directly to compositeness because no coupling suppression was assumed. For leptoquarks, $W^{*}$ bosons etc. the bounds should be about an order of magnitude smaller due to couplings. In the case of new loop effects, those bounds should be further reduced by another order of magnitude. For example, I next consider the effect of heavy $Z^{\prime}$ bosons in loops that enter muon and charged current semileptonic decays differently.

\section{iv) Additional $Z^{\prime}$ Gauge Bosons}

As my final example, I consider the existence of additional $Z^{\prime}$ bosons that influence unitarity at the loop level by affecting muon and semi-leptonic beta decays differently. Such a possibility was considered by Alberto Sirlin and myself [17] about 20 years ago. In general, we found that the unitarity sum was predicted to be greater than one in most scenarios. In fact, one expects

$$
\begin{aligned}
\left|V_{u d}\right|^{2}+\left|V_{u s}\right|^{2}+\left|V_{u b}\right|^{2} & =1+0.01 \lambda \ln X /(X-1) \\
X & =m_{Z^{\prime}}^{2} / m_{W}^{2}
\end{aligned}
$$

where $\lambda$ is a model dependent quantity of $O(1)$. It can have either sign, but generally $\lambda>0$.

In the case of $\mathrm{SO}(10)$ grand unification $Z^{\prime}=Z_{\chi}$ with $\lambda \simeq 0.5$, one finds at one-sided $95 \% \mathrm{CL}$

$$
m_{Z_{\chi}}>560 \mathrm{GeV}
$$

That bound is quite sensitive to the CL used. For example, at one-sided $90 \%$ CL one finds $m_{Z_{\chi}}>1.2$ $\mathrm{TeV}$. Those bounds are similar to tree level bounds on $Z^{\prime}$ bosons from atomic parity violation and polarized Moller scattering [18] as well as the direct collider search bounds [16] $m_{Z_{\chi}}>720 \mathrm{GeV}$.

In addition, interesting unitarity constraints can be placed on supersymmetry [19] where SUSY loops affect muon and semileptonic decays differently. Again, one expects constraints up to mass scales of $O(500 \mathrm{GeV})$, depending on the degree of cancellation between squark and slepton effects.

In the future, the unitarity constraint could improve from \pm 0.0008 to \pm 0.0004 if $f_{+}(0)$ errors and uncertainties from radiative corrections can be reduced. Such an improvement will be difficult, 
but particularly well motivated if the central value of $V_{u s}$ goes down and unitarity appears to be violated. Should that occur, it will be very interesting trying to sort out the source of "new physics" responsible for the deviations and looking for further implications.

\section{References}

[1] N. Cabibbo, Phys. Rev. Let. 10 (1963) 531; M. Kobayashi and T. Maskawa, Prog Theor. Phys. 49 (1973) 652.

[2] W. Marciano and A. Sirlin, Nucl. Phys. B93 (1975) 303.

[3] MuLan Collaboration, D.B. Chitwood et al., Phys. Rev. Lett. 99 (2007) 032001.

[4] W. Marciano and A. Sirlin, Phys. Rev. Lett. 56 (1986) 22.

[5] A. Sirlin, Rev. Mod. Phys. 50 (1978) 573; A. Czarnecki, W. Marciano and A. Sirlin, Phys. Rev. D70 (2004) 093006

[6] G. Savard et al., Phys. Rev. Lett. 95 (2005) 102501; V.E. Iacob et al., Phys. Rev. C74 (2006) 055502; T. Eronen et al., Phys. Rev. Lett. 97 (2006) 232501; P.H. Barker and A.P. Byrne, Phys. Rev. C73 (2006) 064306.

[7] W. Marciano and A. Sirlin, Phys. Rev. Lett. 96 (2006) 032002.

[8] J. Hardy, [hep-ph/0703165].

[9] Flavianet Collaboration, [http: //www. Inf.infn.it/wg/vus]; M Palutan, Kaon07 (2007).

[10] H. Leutwyler and M. Roos, Z. Phys. C25 (1984) 91.

[11] D. Becirevic et al., Nucl. Phys. B705 (2005) 339.

[12] D.J. Antonio et al., [hep-lat/0702026].

[13] V. Cirigliano et al., JHEP 0504 (2005) 006.

[14] E. Gamiz et al., Phys. Rev. Lett. 94 (2005) 011803.

[15] W. Marciano, Phys. Rev. Lett. 93 (2004) 231803.

[16] PDG, W.-M. Yao et al., J. Phys. G33 (2006) 1.

[17] W. Marciano and A. Sirlin, Phys. Rev. D35 (1987) 1672.

[18] P.L. Anthony et al.(SLAC E158 Collaboration), Phys. Rev. Lett. 95 (2005) 081601; A. Czarnecki and W. Marciano, Nature 435 (2005) 43.

[19] R. Barbieri et al., Phys. Lett. 156B (1985) 348; K. Hagiwara et al., Phys. Rev. Lett. 75 (1995) 3605; A. Kurylov and M. Ramsey-Musolf, Phys. Rev. Lett. 88 (2000) 071804. 